\title{
Semi-Empirical Prediction of Aircraft Low-Speed Aerodynamic Characteristics
}

\author{
Erik D. Olson* \\ NASA Langley Research Center, Hampton, VA 23681
}

\begin{abstract}
This paper lays out a comprehensive methodology for computing a low-speed, high-lift polar, without requiring additional details about the aircraft design beyond what is typically available at the conceptual design stage. Introducing low-order, physics-based aerodynamic analyses allows the methodology to be more applicable to unconventional aircraft concepts than traditional, fully-empirical methods. The methodology uses empirical relationships for flap lift effectiveness, chord extension, drag-coefficient increment and maximum lift coefficient of various types of flap systems as a function of flap deflection, and combines these increments with the characteristics of the unflapped airfoils. Once the aerodynamic characteristics of the flapped sections are known, a vortex-lattice analysis calculates the three-dimensional lift, drag and moment coefficients of the whole aircraft configuration. This paper details the results of two validation cases: a supercritical airfoil model with several types of flaps; and a 12-foot, full-span aircraft model with slats and double-slotted
\end{abstract} flaps.

\section{Nomenclature}

$c_{d}, C_{D} \quad$ sectional drag coefficient, total drag coefficient

$c_{d_{p 0}}$

$c_{f}, c_{s}$

$c_{l}, C_{L}$

$c_{\text {max }}, C_{L_{\max }}$

$c_{l_{0}}$

$c_{l_{\alpha}}, C_{L_{\alpha}}$

$c$

$k_{d}$

$k_{s}$

$M$

$R e_{c}$

$\alpha$

$\alpha_{\delta}$

$\delta_{f}, \delta_{s}$

$\Delta_{f}, \Delta_{s}$

$\Delta y$

$\eta_{\delta}$

$\theta_{f}, \theta_{s}$ zero-lift sectional profile drag

trailing-edge flap chord, leading-edge flap chord

sectional lift coefficient, total lift coefficient

maximum sectional lift coefficient, maximum total lift coefficient

sectional lift coefficient at $\alpha=0$

sectional lift-curve slope, total lift-curve slope

section chord

trailing-edge flap profile-drag increment factor

leading-edge flap maximum lift factor

Mach number

Reynolds number based on chord

angle of attack

effective angle of attack factor

trailing-edge flap deflection, leading-edge flap deflection

trailing-edge flap increment, leading-edge flap increment

leading-edge sharpness parameter

trailing-edge flap effectiveness factor

trailing-edge flap chord function, leading-edge flap chord function

\section{Introduction}

CAKEOFF and landing performance requirements often place strong constraints on sizing of the wing and engines during conceptual aircraft design, yet the low-speed aerodynamic characteristics of an aircraft

\footnotetext{
*Aerospace Engineer, NASA Langley Research Center, Senior Member AIAA.
} 
with its flaps deflected and landing gear extended can be difficult to estimate accurately. In particular, the maximum lift coefficient is a difficult value to obtain accurately, even when comprehensive information about the flap and slat geometry is available and the configuration is analyzed using higher-order analysis methods. ${ }^{1,2}$ Traditionally, designers have tended to rely on scaling of available historical data and test results, ${ }^{3}$ but these are of limited use with newer unconventional configurations for which no full-scale data are available. The research described herein seeks to fill this capability gap by providing a comprehensive method for computing a low-speed, high-lift polar without greatly expanding the amount of detail about the aircraft design required to perform the analysis. Introducing low-order, physics-based aerodynamic analyses allows the methodology to be more applicable to unconventional aircraft concepts than traditional, fully-empirical methods.

\section{Methodology}

The methodology for the aerodynamics of an aircraft in the high-lift configuration involves building up the total lift and drag curves in a quasi-three-dimensional manner. First, the shape of the two-dimensional section lift-curve slope is determined from base (unflapped) wing sectional characteristics; second, two-dimensional increments to the section lift-curve slope due to flap deflection are estimated based on information about the flap type and basic geometry; and third, the three-dimensional aerodynamic coefficients of the complete aircraft are determined based on the base and incremental aerodynamic characteristics of the wing sections. The following sections describe these stages in detail.

\section{A. Base Section Aerodynamics}

Figure 1 shows an idealized (linear) section lift curve; in reality the slope of the curve would decrease at higher angles of attack approaching the maximum lift coefficient. However, even though takeoff and landing performance is strongly constrained by the maximum lift coefficient of the aircraft, the actual low-speed operation of the aircraft takes place at more benign lift coefficients where the lift curve is nearly linear. Using this assumption, one can represent the lift curve of the section by three parameters: the lift-curve slope, $c_{l_{\alpha}}$, the angle of attack at zero lift, $\alpha_{l=0}$ (or alternatively, the zero-angle lift coefficient, $c_{l_{0}}$ ), and the maximum lift coefficient, $c_{l \max }$. The DATCOM ${ }^{4}$ provides separate semi-empirical methods for the calculation of these parameters.

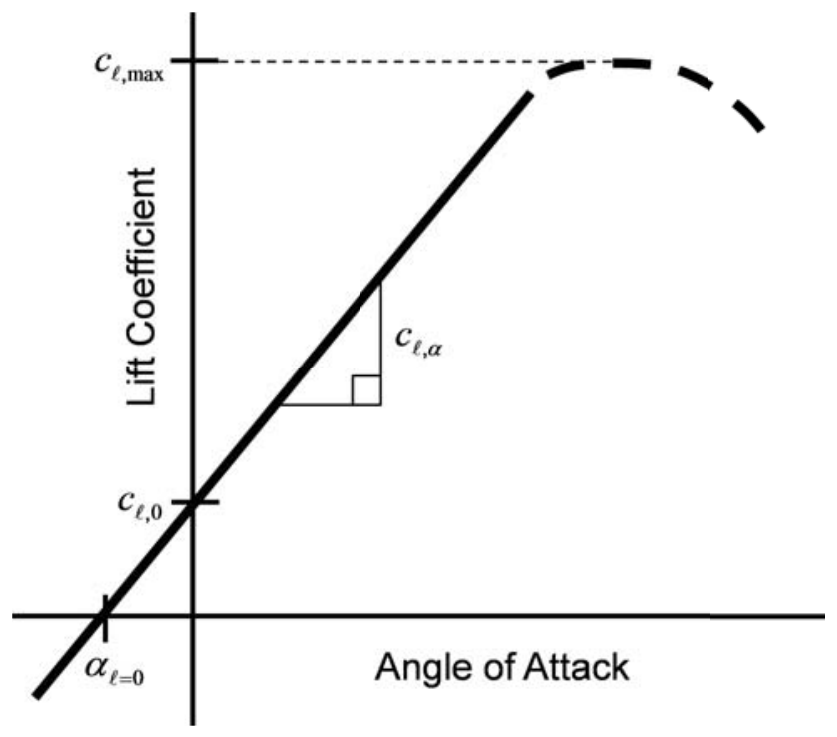

Figure 1. Idealized section lift curve

Alternatively, the section aerodynamics may be determined directly using computational methods. In this study, two different computational methods were evaluated for this purpose: XFoil, ${ }^{5}$ a two-dimensional panel method for single-element airfoils coupled with an integral boundary-layer (IBL) analysis, and MSES, ${ }^{6}$ a two-dimensional Euler analysis for single- and multiple-element airfoils also coupled with an IBL analysis. 
Section III provides a comparison of DATCOM versus computational methods in the analysis of sectional characteristics.

\section{B. Increments Due to Flap Deflections}

When a trailing-edge flap is extended, the following changes occur to the section lift-curve slope, as shown in Fig. 2: the lift at zero angle of attack is increased by an increment $\Delta_{f} c_{l_{0}}$, the lift-curve slope changes to a modified value of $c_{l_{\alpha}}^{\prime}$, and the maximum sectional lift coefficient increases by an increment $\Delta_{f} c_{l \max }$. Methods for estimation of these three effects will be investigated in the following sections. Except where otherwise noted, the methodology in this section follows the approach of Ref. 7.

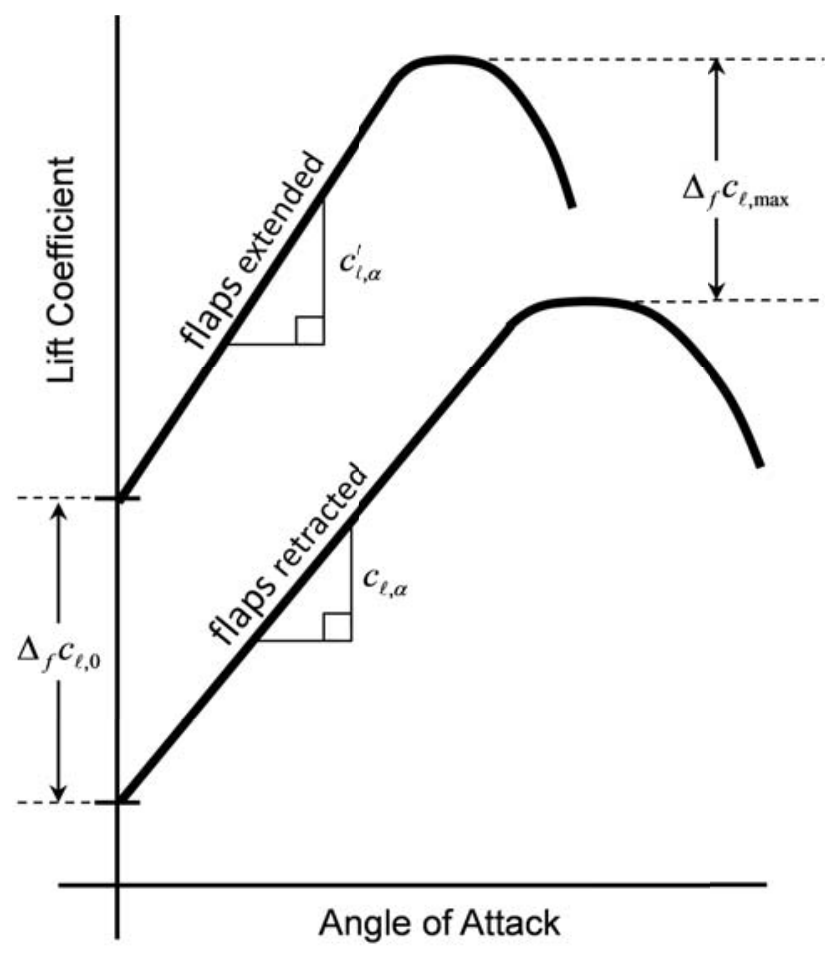

Figure 2. Two-dimensional trailing-edge flap effects

\section{Lift Increment at Zero Angle of Attack}

Flap lift effectiveness is characterized as the change in lift coefficient at zero angle of attack due to flap deflection. Glauert's linearized theory for thin airfoils with flaps ${ }^{8}$ gives the following result for the theoretical increment due to a given trailing-edge flap deflection, $\delta_{f}$ :

$$
\left(\Delta_{f} c_{l_{0}}\right)_{\text {theory }}=\alpha_{\delta} c_{l_{\alpha}} \delta_{f}
$$

where $\alpha_{\delta}$ is the rate of change of zero-lift angle of attack with flap deflection, defined as follows:

$$
\alpha_{\delta} \equiv \frac{c_{l_{\delta}}}{c_{l_{\alpha}}}
$$

where $c_{l_{\delta}} \equiv \frac{\partial c_{l}}{\partial \delta_{f}}$ is the rate of change of lift with flap deflection at constant angle of attack and $c_{l_{\alpha}} \equiv \frac{\partial c_{l}}{\partial \alpha}$ is the rate of change of lift with angle of attack at constant flap deflection. For a thin airfoil, $\alpha_{\delta}$ is related to the flap geometry as follows:

$$
\alpha_{\delta}=1-\frac{\theta_{f}-\sin \theta_{f}}{\pi}
$$

where

$$
\theta_{f}=\cos ^{-1}\left(2 \frac{c_{f}}{c}-1\right)
$$


Here $c_{f}$ is the flap chord.

In practice, the theoretical lift increment in Eq. (1) cannot be realized because of several non-linear effects. First, the small-angle assumption of linear theory breaks down at large flap deflections. Second, the de-cambering effect of the boundary layer near the trailing edge of the flap results in a smaller lift coefficient than predicted. ${ }^{9}$ Finally, at large flap angles the flow can become separated. These effects are captured using an empirical flap effectiveness factor, $\eta_{\delta}$, which is applied to the theoretical equation:

$$
\Delta_{f} c_{l_{0}}=\eta_{\delta} \alpha_{\delta} c_{l_{\alpha}} \delta_{f}
$$

Figure 3 shows empirical curves of flap effectiveness factor for various types of flap systems as a function of deflection angle. These curves allow the conceptual designer to calculate the lift characteristics of representative flap systems under real-world conditions, but they do not require detailed information about the geometry of the flaps.

In addition to changing the camber and incidence of the base airfoil, slotted and Fowler flaps also have the benefit of increasing the effective chord of the section through rearward motion of the flap segments. Since the circulation has a larger chord length to operate on, the flap extension results in an additional increase in lift coefficient proportional to the ratio of the new and old chords. If one assumes that the base section $c_{l_{0}}$ is not altered by the chord extension, the lift coefficient increment with flaps extended can be determined for the extended chord and then referenced against the original chord as follows:

$$
\Delta_{f} c_{l_{0}}=\Delta_{f} c_{l_{0}}^{\prime} \frac{c^{\prime}}{c}+c_{l_{0}}\left(\frac{c^{\prime}}{c}-1\right)
$$

where $c^{\prime} \equiv c+\Delta_{f} c$ is the extended chord and $\Delta_{f} c_{l_{0}}^{\prime}$ is the lift increment referenced against the extended chord:

$$
\Delta_{f} c_{l_{0}}^{\prime}=\eta_{\delta} \alpha_{\delta}^{\prime} c_{l_{\alpha}} \delta_{f}
$$

The value of $\alpha_{\delta}^{\prime}$ is found from Eqs. (3) and (4) by replacing the original flap chord with the extended flap chord, $c_{f}^{\prime} \equiv c_{f}+\Delta_{f} c$. If known, the extended chord can be used directly in Eq. (6), or may be estimated from the empirical curves shown in Fig. 4.

\section{Lift-Curve Slope}

Deflection of trailing-edge flaps has a number of different effects on the lift-curve slope of a section. The potential-flow effect of flap deflection decreases with increasing angle of attack, while viscous effects cause a decrease in flap effectiveness at larger angles of attack. Finally, the chord extension of slotted flaps causes a proportional increase in $c_{l_{\alpha}}$. These effects are complex and difficult to determine accurately; however, a rough estimate of the effects is provided in Ref. 7:

$$
\frac{\left(c_{l_{\alpha}}^{\prime}\right)_{\delta_{f}>0}}{\left(c_{l_{\alpha}}\right)_{\delta_{f}=0}}=\frac{c^{\prime}}{c}\left(1-\frac{c_{f}}{c^{\prime}} \sin ^{2} \delta_{f}\right)
$$

This equation is appropriate for smaller angles of attack $\left(0<\alpha<5^{\circ}\right)$, provided that the slot shape is well optimized and stall has not occurred.

\section{Maximum Lift Coefficient}

The effect of trailing-edge flap deflection on wing maximum sectional lift coefficient is strongly influenced by the stalling behavior of the base airfoil section. This stalling behavior is closely correlated with the leadingedge sharpness parameter, $\Delta y$, which is the difference between the upper-surface ordinates at $6 \%$ chord and $0.15 \%$ chord, respectively, normalized by chord. Airfoils with sharp noses ( $\Delta y$ up to $1.5 \%$ chord) exhibit leading-edge, or long-bubble stall behavior, in which a region of separated flow at the leading edge progresses rearward as the angle of attack is increased, leading to stalling of the entire section. For these sections, thin-airfoil theory finds that the increment in maximum lift coefficient due to flap deflection is related to the theoretical lift increment:

$$
\Delta_{f} c_{l_{\max }}^{\prime}=\left(\Delta_{f} c_{l_{0}}^{\prime}\right)_{\text {theory }} \frac{\sin \theta_{f}}{\pi-\left(\theta_{f}-\sin \theta_{f}\right)}
$$




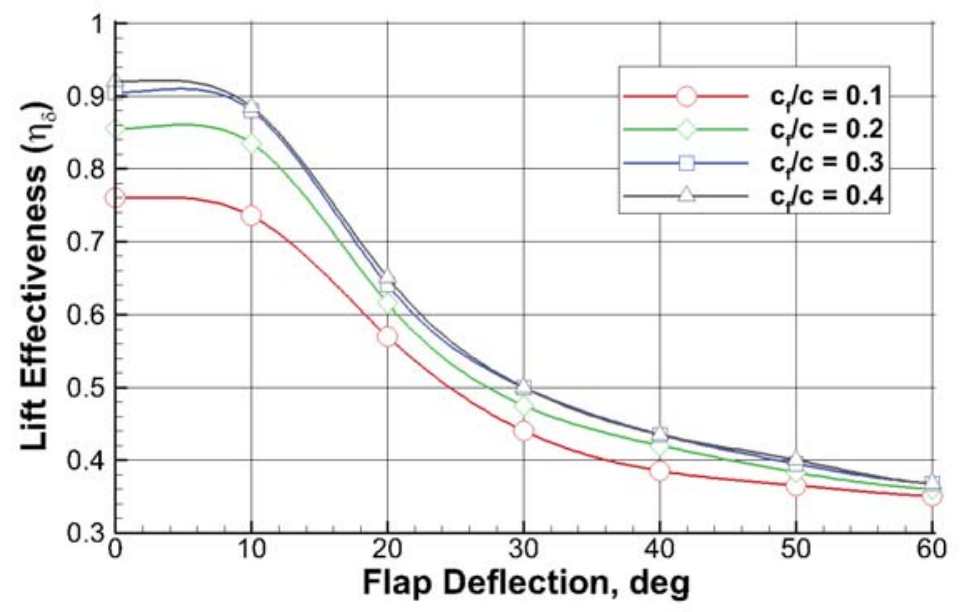

(a) Plain flaps with sealed gap

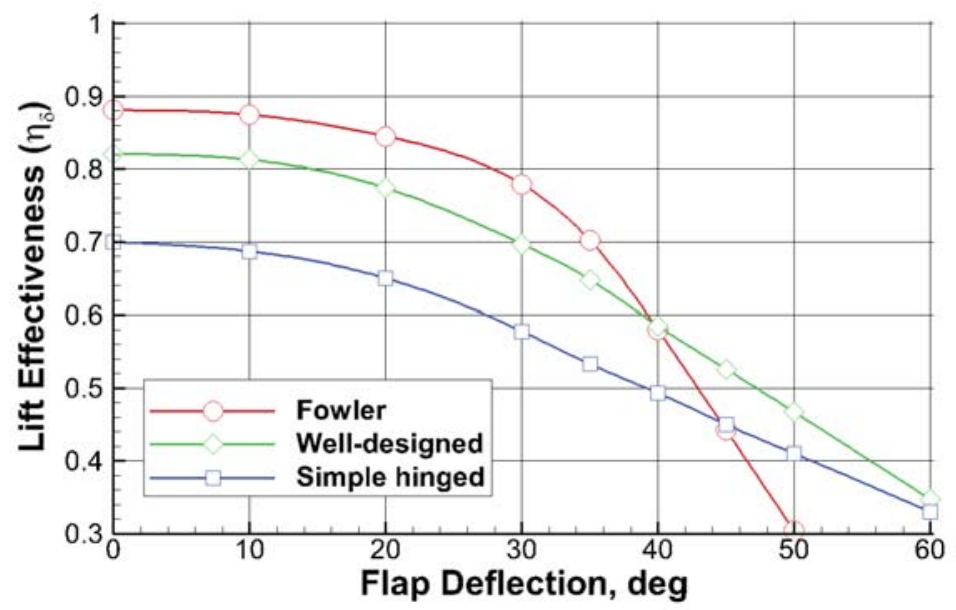

(b) Single-slotted flaps

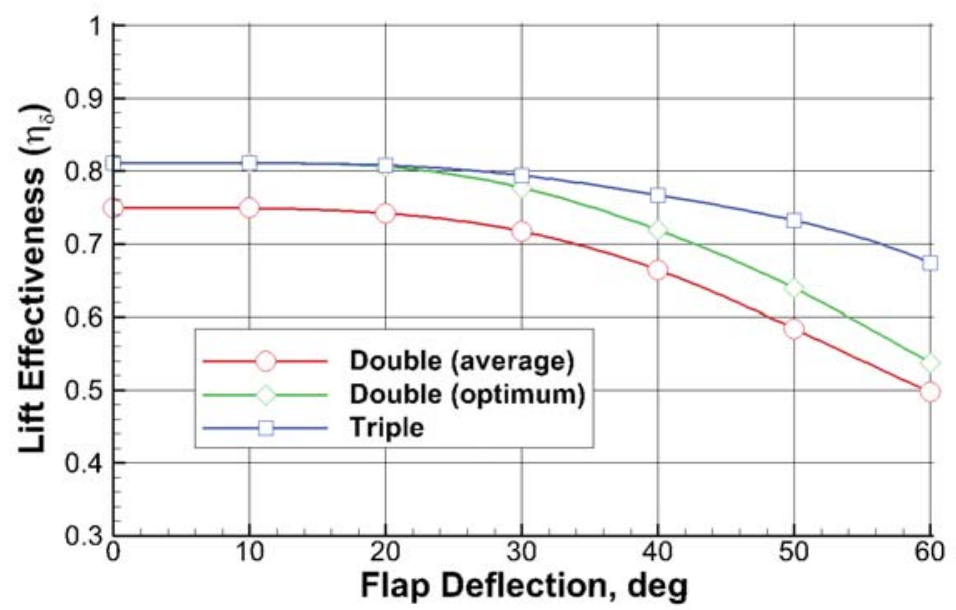

(c) Double- and triple-slotted flaps

Figure 3. Empirical lift effectiveness, $\eta_{\delta}$ for various types of trailing-edge flaps as a function of deflection angle. Source: data from Ref. 7. 


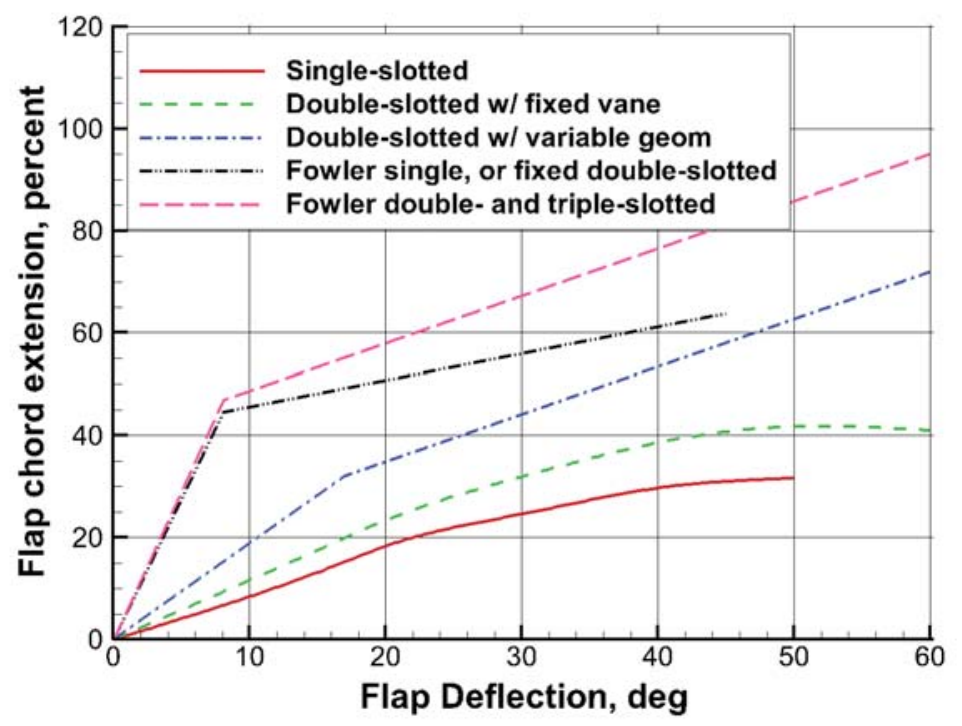

Figure 4. Flap chord extension as a function of deflection for different flap types. Source: data from Ref. 7.

For practical ranges of flap chord ratio, Eq. (9) results in a $\Delta_{f} c_{l \max }^{\prime}$ which is about half of the theoretical lift increment. Combining Eq. (9) with Eqs. (4) and (7) yields the following simple expression for the maximum lift increment for airfoils with sharp noses $(\Delta y<1.5)$ :

$$
\Delta_{f} c_{l_{\max }^{\prime}}^{\prime}=c_{l_{\alpha}} \delta_{f} \frac{\sin \theta_{f}}{\pi}
$$

As $\Delta y$ increases above 1.5, the section displays a short-bubble stalling behavior and the maximum lift increment increases approximately proportionally with $\Delta y$ to the point where the stall is dominated by trailing-edge stall. Beyond this value of $\Delta y$, the base section displays a separation behavior that is associated with the pressure gradient near the trailing edge; therefore, the stall will be delayed by the local suction produced by the initial deflection of a trailing-edge flap. ${ }^{10}$ Beyond a certain flap angle, however, the load induced at the airfoil nose will be high enough that stall will occur first at the leading edge and the maximum lift increment will behave in a manner similar to the sharp-nose airfoil relationship in Eq. (10).

The maximum lift coefficient for airfoils with short-bubble or trailing-edge stalling behavior $(\Delta y \geq 1.5)$ may thus be determined by finding which of the two stalling mechanisms is dominant:

$$
c_{l_{\max }}^{\prime}=\min \left(\begin{array}{c}
\left(c_{l_{\max }}\right)_{\delta_{f}=0}+\Delta_{f} c_{l_{0}}^{\prime} \\
0.533 \frac{\Delta y}{c}\left(\frac{R e_{c}}{3 \times 10^{6}}\right)^{0.08}+\frac{1}{2}\left(c_{l_{0}}+\Delta_{f} c_{l_{0}}^{\prime}\right)
\end{array}\right)
$$

The top term in Eq. (11) reflects the one-to-one relationship between the lift increment and the increase in maximum lift for airfoils experiencing trailing-edge stall at smaller flap deflections, whereas the lower term reflects the increase in maximum lift for airfoils experiencing leading-edge stall at larger flap deflections. Ref. 7 suggests that this equation can be expected to give acceptable results for both plain and slotted flaps, including multi-element flaps with or without Fowler motion.

\section{Profile-Drag Increment}

Basing $\Delta_{f} c_{l}$ on Glauert's linear airfoil theory and accounting for chord extension, the minimum-profile-drag increment can be expressed as

$$
\Delta_{f} c_{d_{p 0}}=k_{d} c_{l_{\alpha}} \alpha_{\delta}^{\prime} \frac{c_{f}}{c} \delta_{f} \sin \delta_{f}+c_{d_{p 0}}\left(\frac{c^{\prime}}{c}-1\right)
$$

where $\alpha_{\delta}^{\prime}$ is defined as before. The profile-drag factor $k_{d}$ is an empirical function of the type of flap and the deflection angle, as shown in Fig. 5. This figure shows that the actual drag increment is on the order of 20 


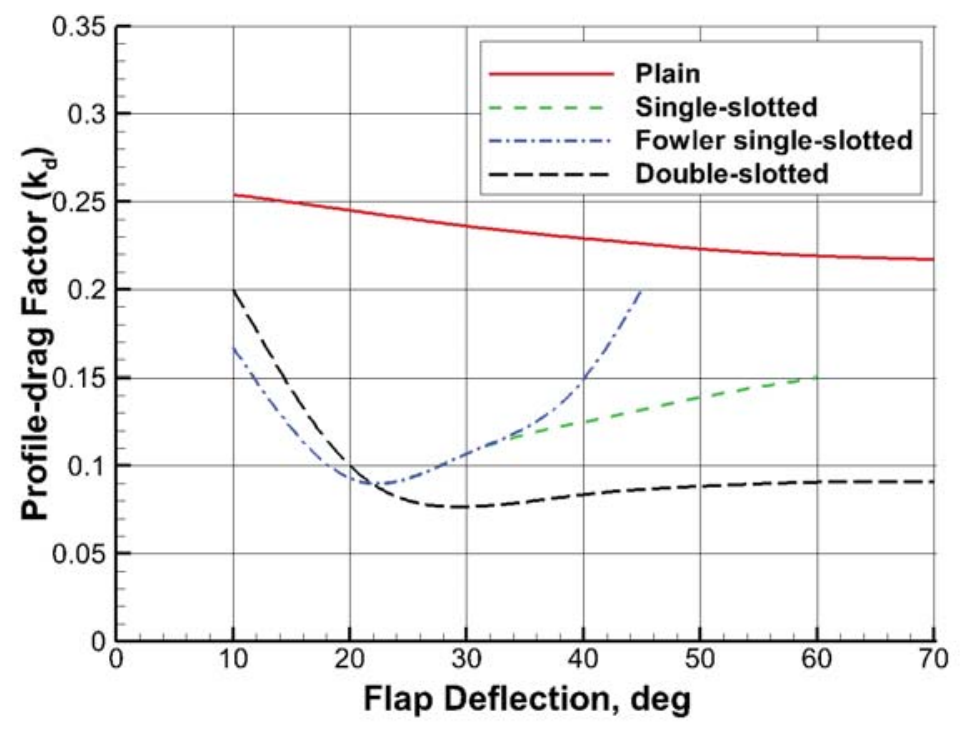

Figure 5. Effect of trailing-edge flap deflection on profile drag factor, $k_{d}$. Source: data from Ref. 7 .

to $25 \%$ of the theoretical drag increment for plain flaps, and 8 to $15 \%$ for slotted flaps. The high value of $k_{d}$ for slotted flaps at small deflection angles is due to unfavorable slot flow and depends to a large extent on the details of the flap system design.

\section{Effects of Leading-Edge Flaps}

Even airfoils with rounded noses will exhibit leading-edge stall at larger trailing-edge flap deflection angles. When leading-edge devices (either flaps or slats) are deployed, the high suction peak near the leading edge of the airfoil is alleviated, delaying the leading-edge separation to a higher angle of attack and increasing the maximum lift coefficient. ${ }^{10}$ However, a slat wake may interfere with the flow around trailing-edge flaps and the increment in $c_{l_{\max }}$ is up to $15 \%$ less than for the undeflected case.

Leading-edge droop causes a loss in lift at zero angle of attack which can be derived from Glauert's thin airfoil theory:

$$
\Delta_{s} c_{l_{0}}=-\frac{\theta_{s}-\sin \theta_{s}}{\pi} \delta_{s} c_{l_{\alpha}}
$$

where

$$
\theta_{s}=\cos ^{-1}\left(1-2 \frac{c_{s}}{c}\right)
$$

If one assumes that the loss of lift is entirely due to a change in the ideal angle of attack of the section-that is, the angle at which the flow approaches the leading edge of the airfoil smoothly and causes no extreme suction peak at the nose - then the change in maximum lift coefficient can be derived from DATCOM data to give

$$
\Delta_{s} c_{\max }=0.58 \sqrt{\frac{c_{s}}{c}} \delta_{s} c_{l_{\alpha}}
$$

This relationship is valid for deflection angles up to approximately 25 degrees. Leading-edge flap deflection has no appreciable effect on the lift-curve slope provided that the flap is not deflected much more than 20 degrees.

As in the case of plain leading-edge flaps, extension of a slat results in a loss of lift at zero angle of attack. However, a slat also increases the effective chord length; both effects are of the same order of magnitude and one may assume that they cancel each other out. Assuming that the trailing-edge flap and the slat have been designed together so that all parts of the system experience separation at nearly the same angle of attack, the following expression may be used for the airfoil with slats deployed:

$$
c_{l_{\max }}=\left(1-k_{s}\right) \frac{c_{l_{0}}+\Delta_{f} c_{l_{0}}+0.47 c_{l_{\alpha}}}{1+0.035 c_{l_{\alpha}}}
$$


where $k_{s}$ is a factor taking into account the non-linearity of the lift curve at high lift and generally varies between 0.03 and 0.15 , with 0.07 as a reasonable average. The maximum lift of Krueger flaps is very similar to that of slats and Eq. (16) may be used in this case as well.

\section{Three-Dimensional Aerodynamics}

The final step in the low-speed analysis is to calculate the three-dimensional aerodynamic coefficients of the complete aircraft based on the base and incremental aerodynamic characteristics of the wing sections. In this methodology the Athena Vortex-Lattice (AVL) computer program ${ }^{11}$ is used. Given the two-dimensional characteristics of the wing and tail sections, AVL can be run with either an ideal lift curve slope $\left(c_{l_{\alpha}}=2 \pi \alpha\right)$ or with a lift-curve slope determined from DATCOM or from two-dimensional computational analysis; the effect of this choice is examined in Section III.

AVL models all lifting surfaces as "flat"; all sections of a surface are parallel to the $x$-axis, and the incidence and camber of lifting surfaces are modeled as a tilting of the control point normal vectors rather than being reflected in the physical shape of the surface. Along these lines, the deflection of control surfaces (such as flaps, elevators, and ailerons) is also modeled as a tilting of control point normal vectors rather than a physical deflection of the surface. AVL is a linear code, so flap deflections produce lift increments in a manner consistent with Eq. (1). To account for the non-linear effects discussed previously, the empirical lift effectiveness factor, $\eta_{\delta}$ from Eq. (5), is determined for each flap component and input to AVL as the ratio between the effective and actual flap deflections, referred to in the program as flap gain. The increment in zero-lift drag is also added to the section information, while the increment in pitching-moment is calculated by AVL.

The chordwise extension of slotted flaps is modeled as a physical chord increase in the AVL geometry, but for calculation of aerodynamic coefficients the reference area remains unaffected. This chord extension can come from either historical data for different flaps, as seen in Fig. 4, or from the actual chord extension of the flap, if known. Since in this methodology slats have no influence on lift, they are not modeled in the AVL geometry, but are factored into the zero-lift drag of the section and are used in the calculation of maximum sectional lift coefficient.

In addition to the lift-curve slope, the zero-lift drag coefficient from DATCOM and the flap drag increment from Eq. (12) are input to AVL for each section of the wing and tails, with the program automatically integrating the values to determine the total profile drag of each lifting surface. The methodology calculates the drag of the fuselage using the FRICTION computer program ${ }^{12}$ - which determines the profile drag of aircraft components using empirical skin-friction drag of a flat plate combined with empirical form factorsand adds it to the drag of the lifting surfaces to arrive at the drag of the complete aircraft.

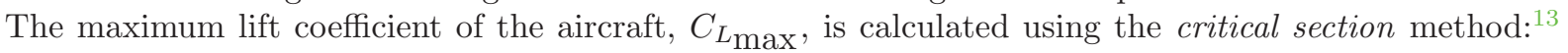
for increasing values of angle of attack, the lift coefficient at each location along the wing is compared to the maximum lift coefficient of that section and a stall is declared when the maximum lift coefficient is first surpassed at any section. The maximum lift coefficient for the aircraft is defined as the value achieved when the stalling condition is reached. The critical section method is simple and computationally inexpensive; for a highly-swept wing, however, the actual $C_{L \max }$ tends to be lower due to the effects of spanwise flow at higher angles of attack, and studies have found that the critical section method can be optimistic in these cases. $^{14}$

\section{Validation}

Validation of the methodology was performed for two different cases. First, a supercritical airfoil model was used to test the validity of the two-dimensional aerodynamic analysis of the base section and associated increments due to flap and slat deflection. Second, a full-span, high-aspect-ratio transport model was used to test the validity of the three-dimensional aerodynamic analysis.

\section{A. Supercritical Airfoil with Flaps}

The two-dimensional analysis methodology was validated using experimental results for a 9.3\%-thick supercritical airfoil. ${ }^{15,16}$ Test results are available for this airfoil for four different geometric configurations: the base airfoil without flaps or slats installed, and the same airfoil configured with a single-slotted flap, a 
double-slotted flap and slat combination, and a triple-slotted flap and slat combination. In this study the first three configurations were analyzed.

Figure 6(a) shows analysis results for the base airfoil using three different methods: DATCOM empirical predictions, and MSES and XFoil analyses. The DATCOM predictions show good agreement for the liftcurve slope of the airfoil but under-predict the zero-angle lift coefficient and the maximum lift coefficient. The DATCOM database is based on earlier airfoil designs, and newer supercritical airfoils tend to fall outside the range of some of the correlating parameters, due in particular to the large amount of aft camber. The estimate of zero-lift drag compares favorably with the experimental data, but DATCOM does not provide methods for profile drag-due-to-lift or moment coefficient so those comparisons are not included.

Both MSES and XFoil give accurate estimates of the lift curve, drag polar and moment-coefficient curves for the base airfoil. XFoil, being a panel code, is robust at high angles of attack and gives results up to and past stall, whereas MSES tends to only return results up to the vicinity of the stall. Note that the data points in Fig. 6(a) do not show a clear stalling point, but the final data point exhibits a rapid drag increase and appears to be close to the stall. If this is true, XFoil estimates maximum lift coefficient accurately for this case, whereas MSES slightly under-predicts it.

Analysis of the airfoil with a single-slotted flap was performed using XFoil results for the base airfoil combined with the empirical flap increments described previously, as well as using direct analysis of the airfoil and flap geometry in MSES (Fig. 6(b)). Both the empirical curves and MSES give good results for the lift increment due to flap deflection. Both methods predict a maximum lift coefficient just over 2.5 but again the data do not indicate a clear stalling point so the experimental maximum lift coefficient is unknown. The drag coefficient increment added to the XFoil analysis gives a reasonable estimate of the total drag with the flap deflected, but since it is not a function of angle of attack the shape of the drag polar differs from the experimental results. Both analysis methods over-estimate the nose-down pitching-moment increment of the trailing-edge flap deflection. The ability of the two-dimensional methods to calculate the moment-coefficient increment, however, is not relevant here because this increment is calculated by AVL in the full methodology.

Finally, the airfoil with double-slotted flap and slat was analyzed using XFoil only, combined with the empirical flap increments, since MSES would not converge for this case (Fig. 6(c)). The empirical curves give an accurate estimate of the lift increment, but somewhat overestimate the drag increment due to flap deflection. Contrary to the single-slotted flap case, the moment-coefficient increment is greatly underestimated for the double-slotted case.

Table 1 gives a summary of the maximum lift coefficient calculated by the different methods compared to the experimental results for each case. The " $\geq$ " symbol indicates values for which the actual stall point is not clear from the data. The combination of XFoil with empirical flap increments provides reasonably accurate results for cases where the maximum lift coefficient can be determined from the data.

Table 1. Maximum lift coefficient results-9.3\% supercritical airfoil, Mach $\mathbf{0 . 2 0 1}, \operatorname{Re}_{c}=2.83 \times 10^{6}$

\begin{tabular}{|l|r|r|r|}
\hline Analysis method & Base airfoil & Single-slotted & Double-slotted w/ slat \\
\hline DATCOM & 1.53 & - & - \\
MSES & $\geq 1.61$ & 2.57 & - \\
XFoil & 1.74 & 2.51 & 4.49 \\
Data & 1.72 & $\geq 2.42$ & $\geq 4.57$ \\
\hline
\end{tabular}

\section{B. EET AR12 Model}

As a validation case for the three-dimensional analysis of an aircraft with flaps, the EET AR12 $2^{17,18}$ model was used. This model is a 12-foot full-span aircraft configuration with a supercritical wing, full-span slats, and part-span double-slotted flaps with a cutout for the engine (Fig. 7). The model also can be equipped with a movable horizontal tail, flow-through nacelles, and landing gear, but these were not installed for the test cases used in this study.

An OpenVSP ${ }^{19}$ model of the EET AR12 was constructed using the published planform shape, airfoil coordinates, twist distribution, and fuselage cross-section shapes (Fig. 8). The OpenVSP model was then converted to an AVL model using an in-house automated conversion routine; flap and slat definitions were added to the AVL model during this conversion process. Since both the chord extension and flap gain are 


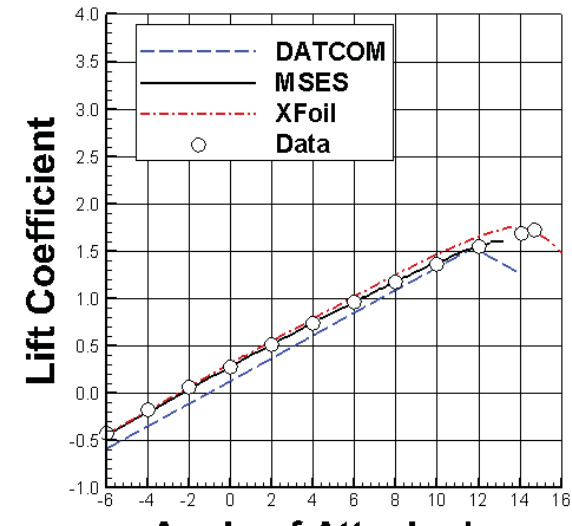

Angle of Attack, deg

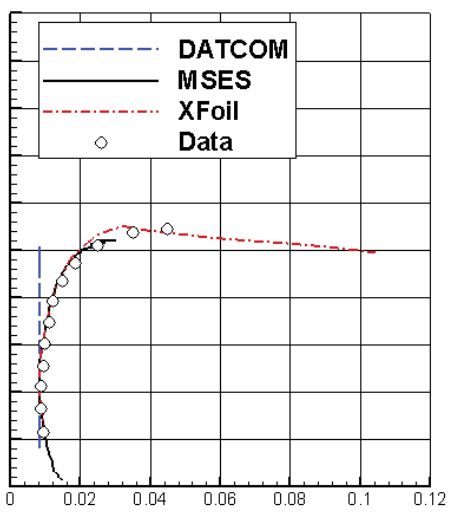

Drag Coefficient

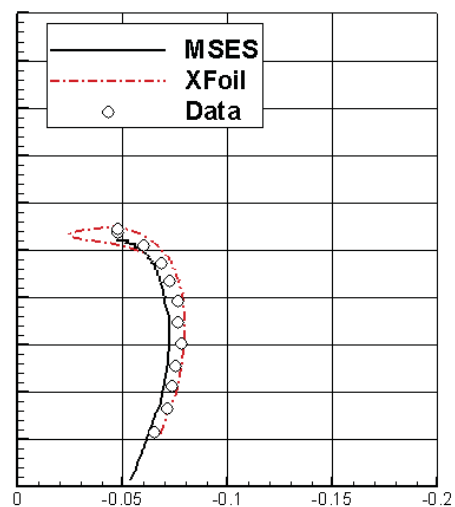

Moment Coefficient

(a) Base airfoil

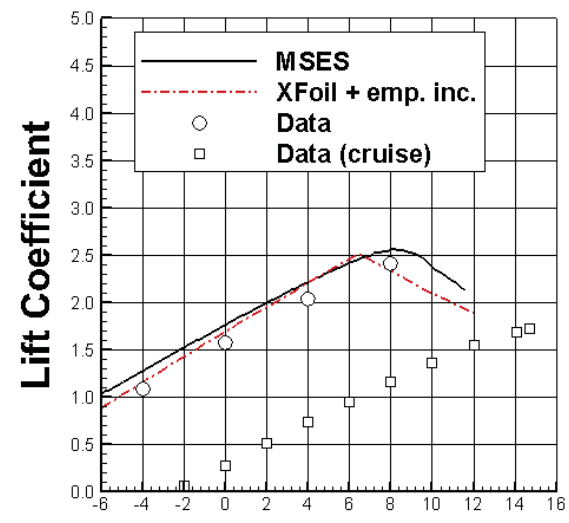

Angle of Attack, deg

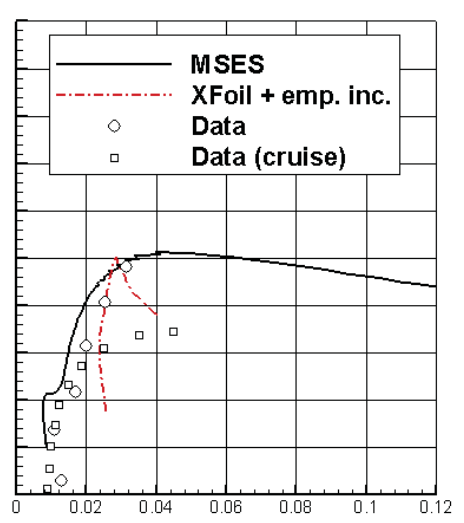

Drag Coefficient

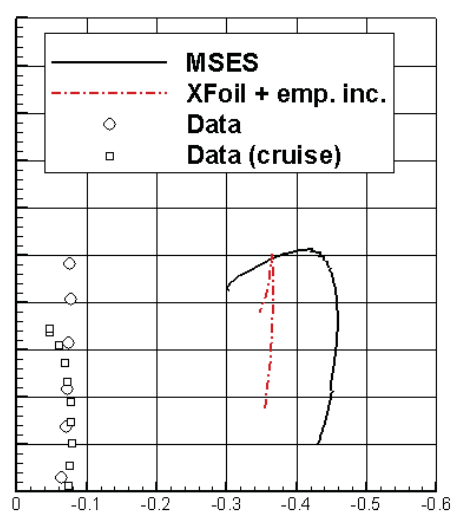

Moment Coefficient

(b) Single-slotted flap, $\delta_{f}=20^{\circ}$

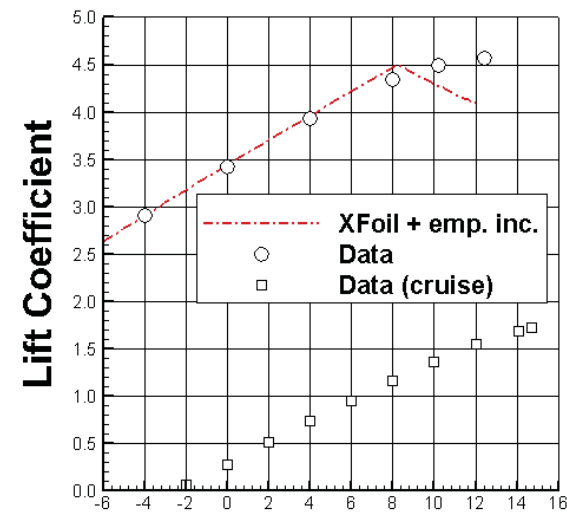

Angle of Attack, deg

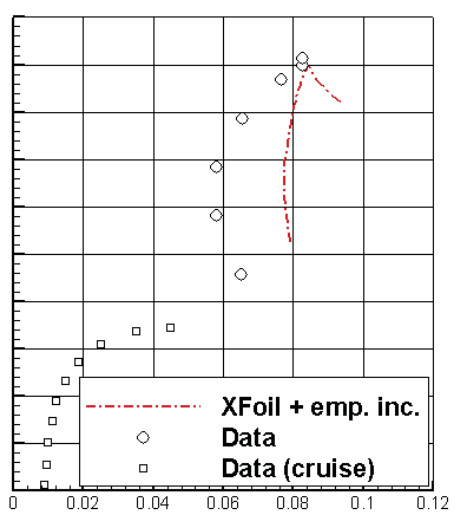

Drag Coefficient

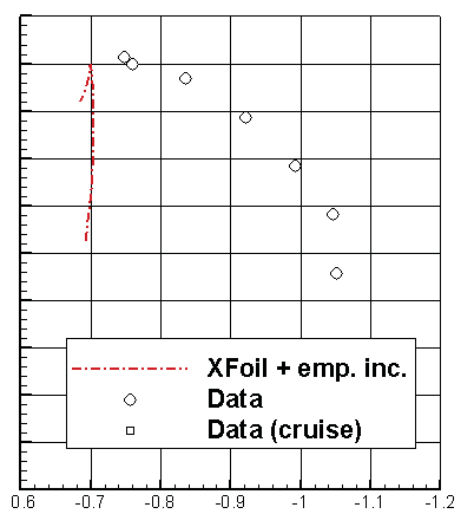

Moment Coefficient

(c) Double-slotted flap with slat, $\delta_{s}=45^{\circ}, \delta_{f}=44^{\circ}$

Figure 6. Analysis results-9.3\% supercritical airfoil, Mach $\mathbf{0 . 2 0 1 ,} R e_{c}=2.83 \times 10^{6}$ 

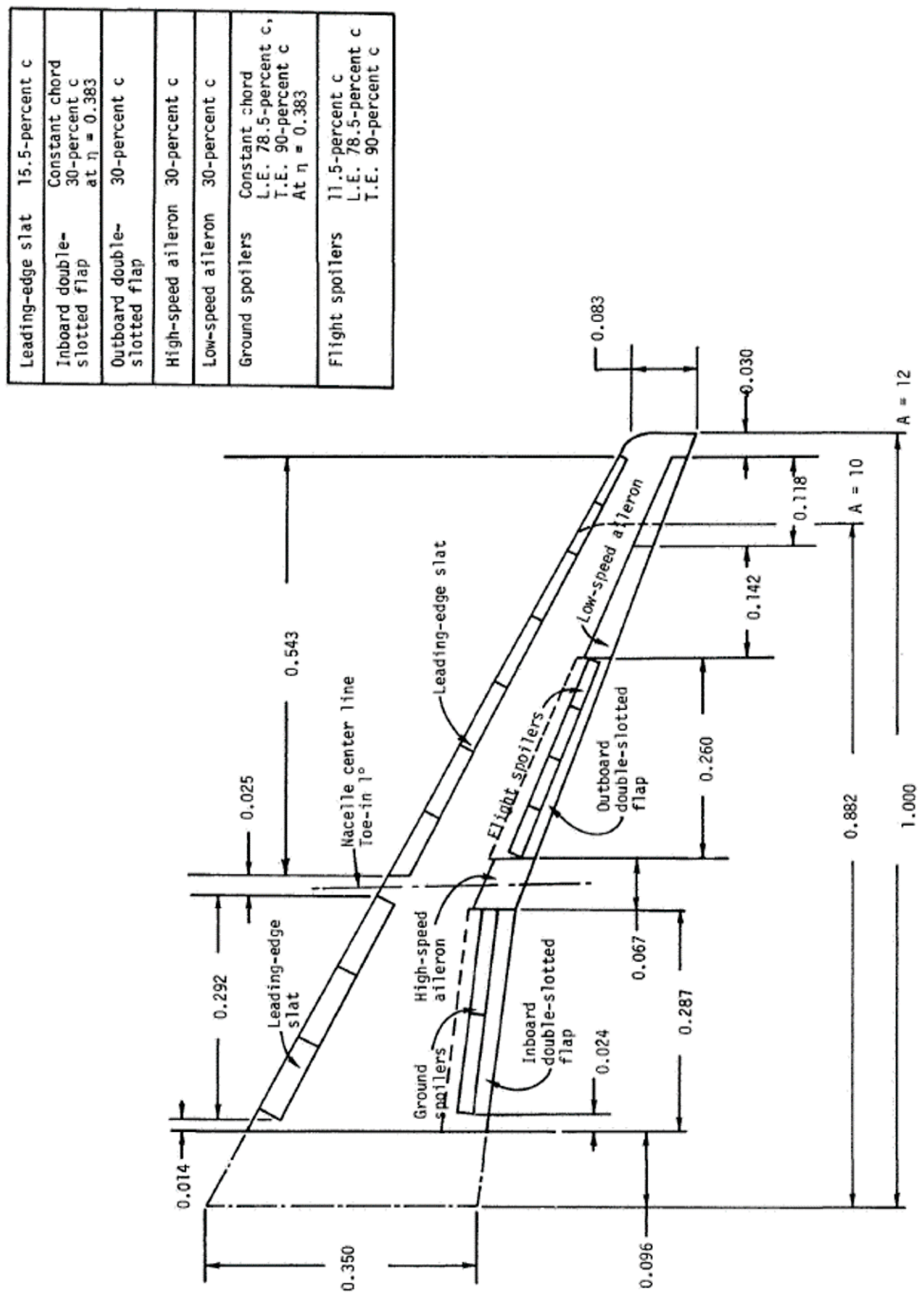

Figure 7. EET AR12 model planform detail. Source: Ref. 18

\section{1 of 16}




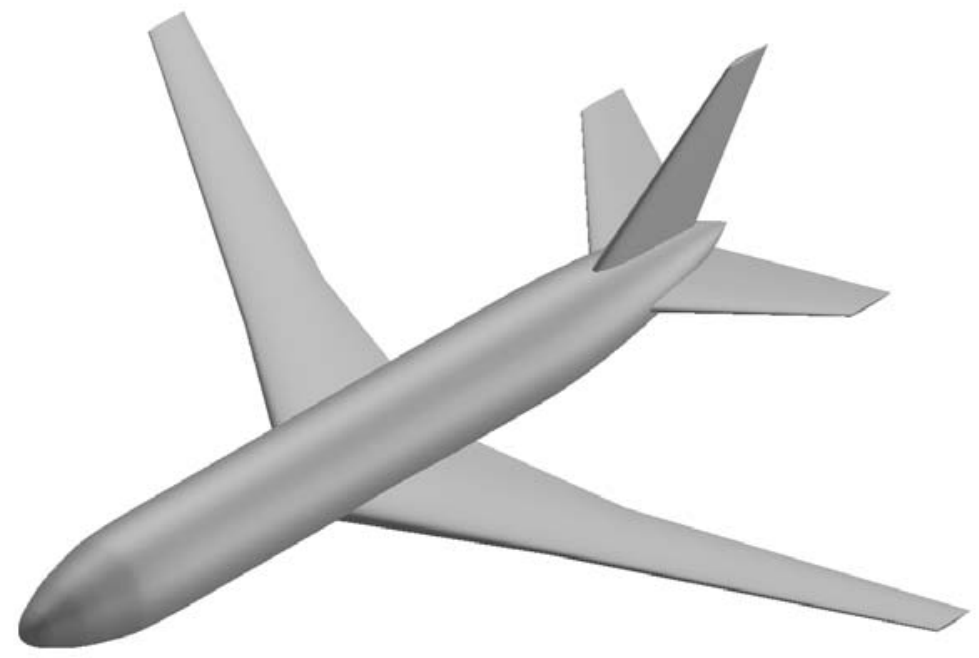

Figure 8. EET AR12 OpenVSP model

functions of the trailing-edge deflection, separate AVL models were required for the cruise wing (flaps stowed) and the takeoff and landing configurations, as illustrated in Fig. 9. The AVL model uses a cruciform fuselage model with no wake shedding that produces a moment in both the longitudinal and directional axes but near-zero lift and side forces for the fuselage. The wing surface includes a connecting section that carries the lift from the side of body across the fuselage centerline but is uncambered to correct for the poor lifting efficiency of the fuselage. The combination of a non-lifting fuselage with a wing carry-through was found to produce the best match between the predicted and experimental lift curves compared to other modeling strategies.

Validation results are shown in Fig. 10 for the three cases: cruise, takeoff and landing. In each case the incidence angle of the horizontal tail was kept fixed at zero degrees. Since the vortex-lattice analysis is linear, the lift curves do not capture the effects of stall. However, the maximum lift coefficient of the configuration was calculated using the critical section method, with the overall lift curve calculated by the combination of DATCOM plus AVL, and with the sectional maximum lift coefficients calculated by XFoil. Table 2 compares the results of this analysis with experimental measurements.

Table 2. Maximum lift coefficient results-EET AR12 model, Mach $\mathbf{0 . 1 6 8 ,} R e_{c}=1.37 \times 10^{6}$

\begin{tabular}{|l|r|r|r|}
\hline Analysis method & Cruise wing & Takeoff flaps & Landing flaps \\
\hline AVL + DATCOM + XFoil & 1.79 & 2.41 & 2.77 \\
Data & 1.34 & 2.51 & 2.82 \\
\hline
\end{tabular}

For the cruise wing (Fig. 10(a)), the lift curve and drag polar match well in the pre-stall region. The pitching-moment prediction is not as good and there is significant difference in the slope of the curve compared to experimental results. The maximum lift coefficient is over-predicted by $33 \%$, but as noted previously the critical section method can be expected to be optimistic for a swept wing.

For the takeoff configuration (Fig. 10(b)), the predicted lift curve matches well at moderate angles of attack, but there is a much larger difference at lower angles of attack. In the experimental data there is an unexpectedly low lift increment and non-linearity in the lift curve at near-zero angles of attack, suggesting that the combination of takeoff flap geometry and deflection was not particularly ideal at those conditions. The differences between the predicted and experimental lift curves in this region can therefore be expected, because the empirical curves for flap effectiveness assume a well-optimized flap design. The drag polar underestimates the induced-drag effects of the flap deflection, but the method gives a reasonable approximation of the drag coefficient at moderate angles of attack. The prediction of maximum lift coefficient compares favorably with the data. At moderate angles of attack, the slope of the pitching-moment curve is inaccurate 


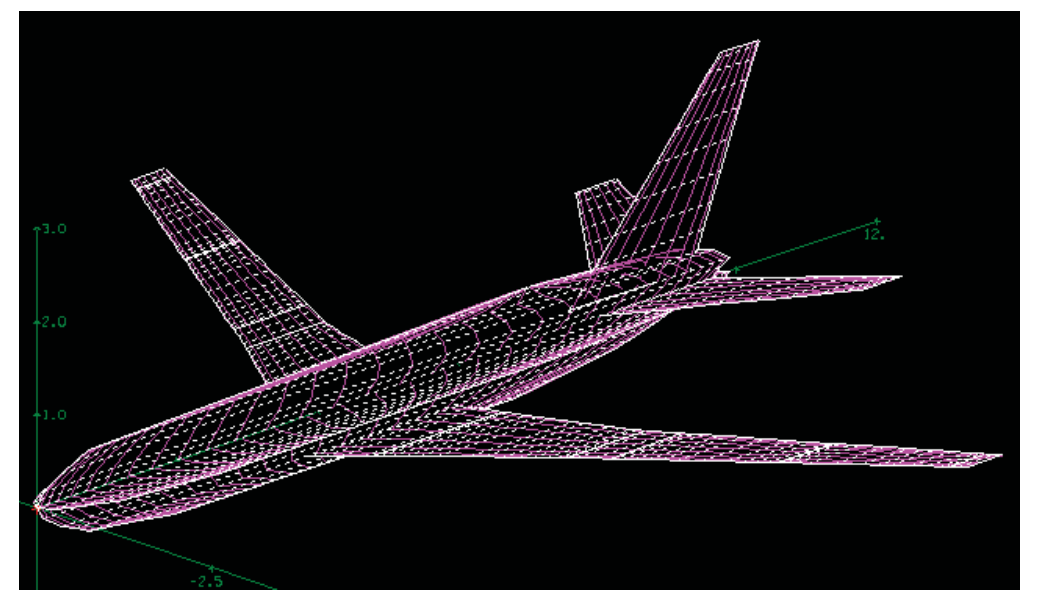

(a) Cruise wing

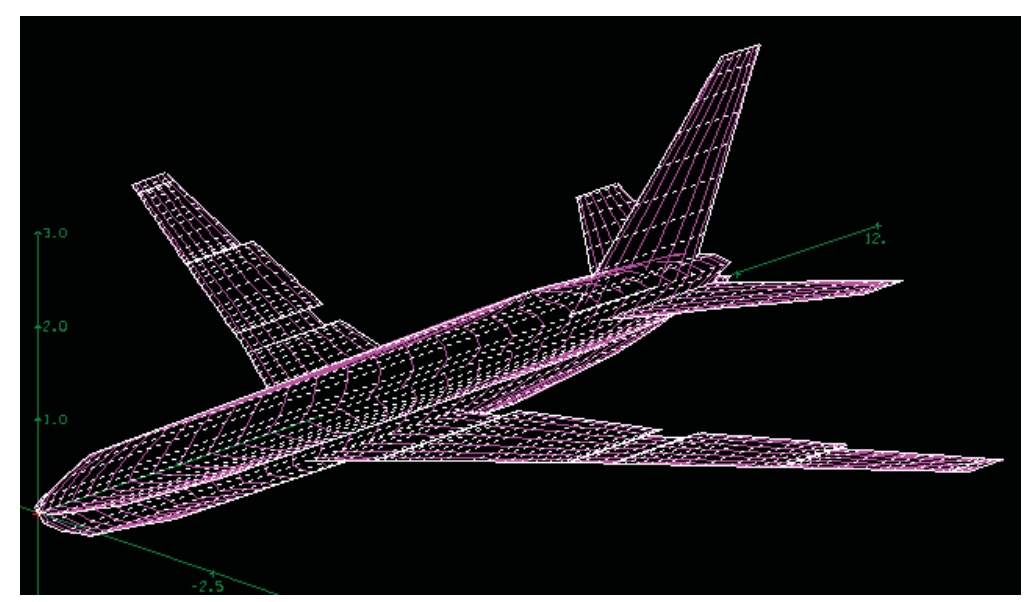

(b) Takeoff wing

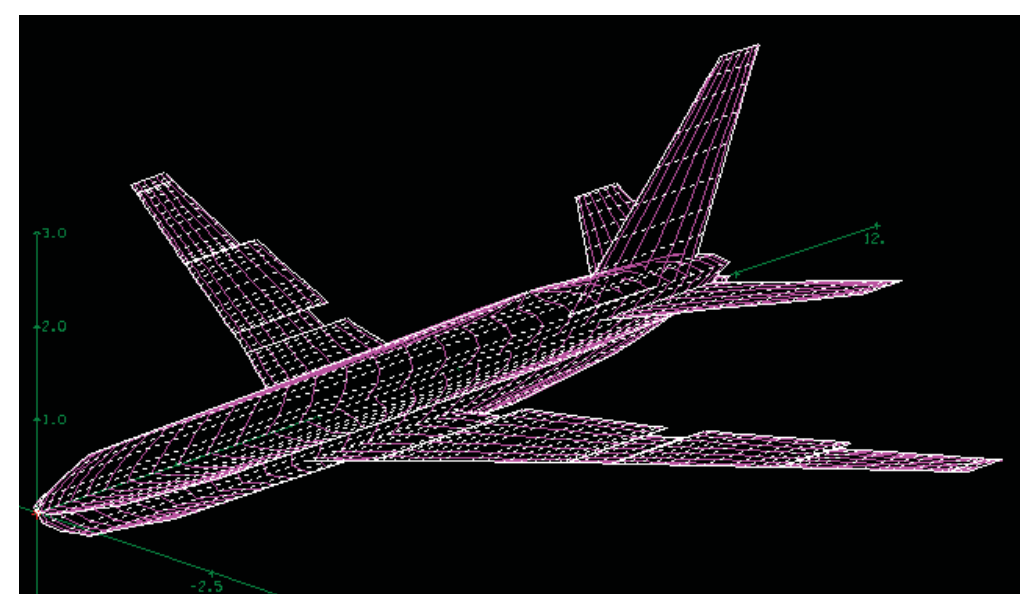

(c) Landing wing

Figure 9. EET AR12 AVL models for different flap settings 

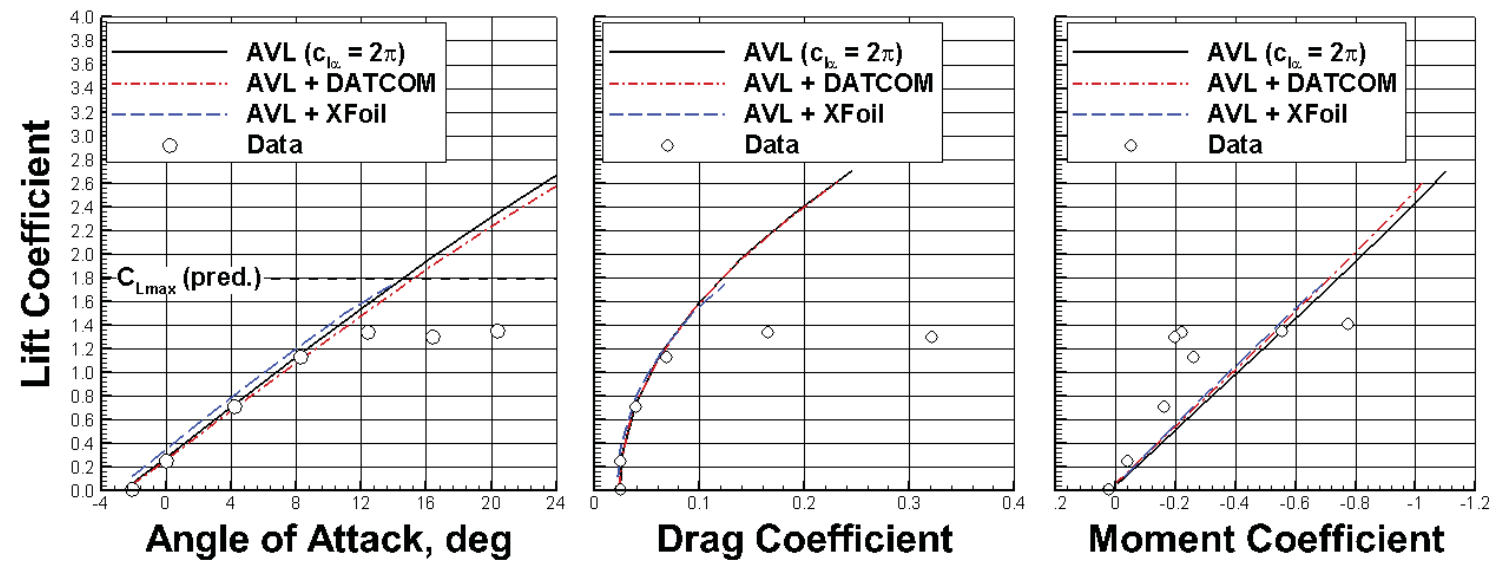

(a) Cruise wing
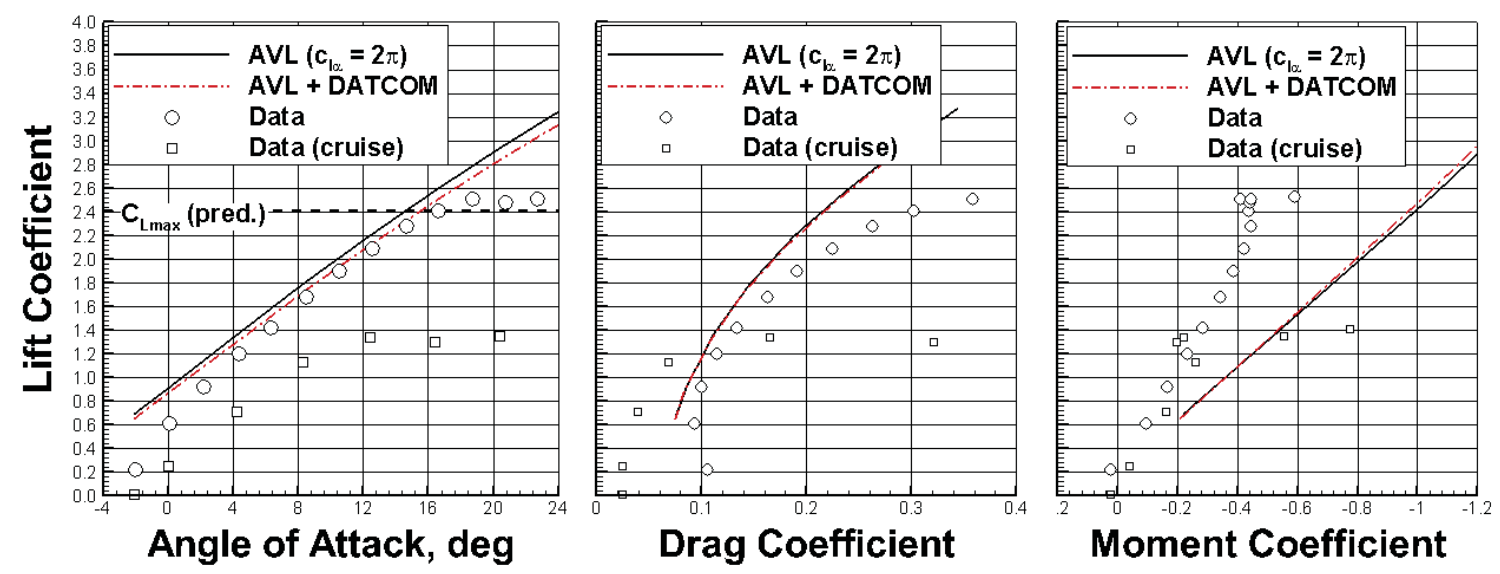

(b) Takeoff flaps, $\delta_{s}=50^{\circ}, \delta_{f}=30^{\circ}$
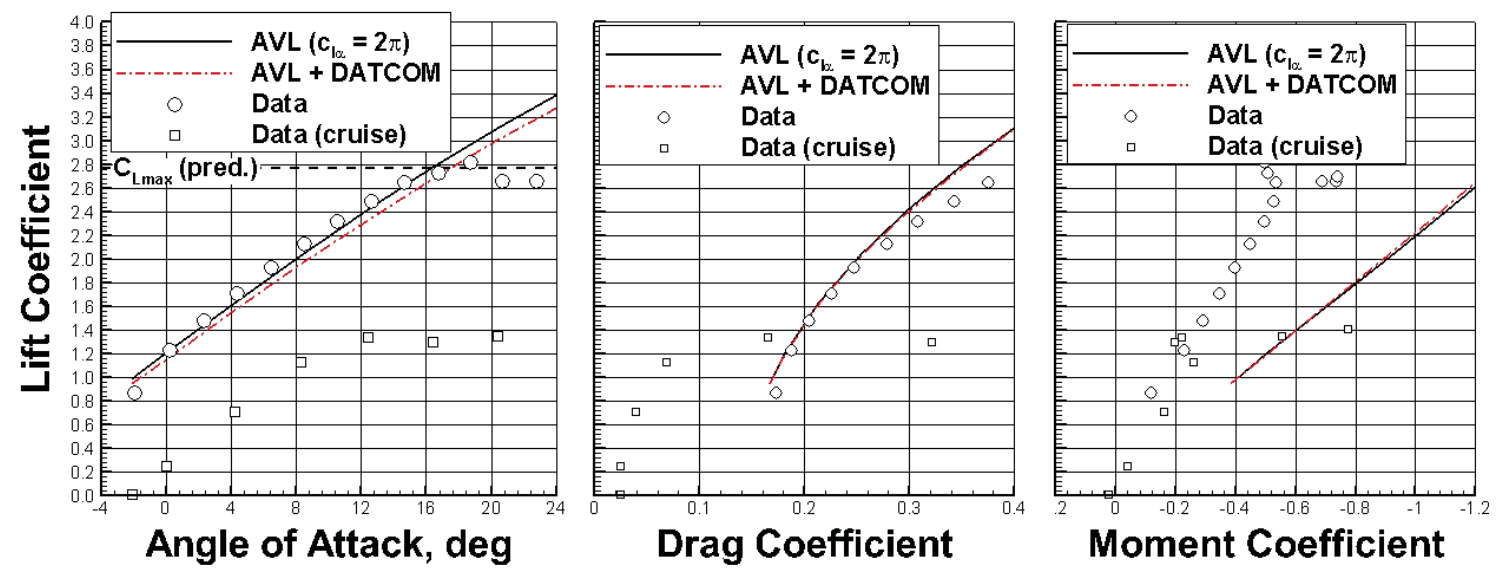

(c) Landing flaps, $\delta_{s}=50^{\circ}, \delta_{f}=60^{\circ}$

Figure 10. Analysis results-EET AR12 model, Mach 0.168, $R e_{c}=1.37 \times 10^{6}$ 
despite the lift and drag being well-predicted.

The landing configuration comparisons are shown in Fig. 10(c). Again, the lift curve and drag polar show good comparison with experimental results, and the prediction of maximum lift coefficient compares favorably with the experimental value. As before, the magnitude of the slope of the pitching-moment curve is significantly over-predicted relative to the data.

\section{Conclusion}

The classical DATCOM predictions proved to be somewhat inadequate for the prediction of the lift curve and maximum lift coefficient of the airfoils used in this study. The predictions are based on empirical curve fits to airfoil existing at the time, and newer supercritical airfoil designs are not part of the database. Shape parameters for supercritical airfoils, particularly the location of the maximum camber, are not properly captured. Analysis of sectional aerodynamics using XFoil produced good agreement with experimental data; being a panel method, it is very robust at high angles of attack and predicts $c_{l \text { max }}$ fairly well with the caveat that the onset of stall tends to occur more gradually than in the experimental data. Analysis with MSES also produced good agreement with experimental data but it was more difficult to produce converged solutions at high angle of attack in order to capture the stall point.

For prediction of the aerodynamics of the full aircraft in the cruise configuration, vortex-lattice analysis compared favorably with experimental data. The lift curve was accurately modeled although the predicted $C_{L \max }$ was optimistic; this was as expected due to the importance of spanwise flow near stall for a swept wing. Both the zero-lift drag coefficient and the induced drag were matched well. The moment coefficient of the full configuration at zero lift was fairly accurate but the slope of the moment coefficient curve with angle of attack was not well predicted.

For the takeoff flap setting, the increment due to flap deflection was predicted well except at low angles of attack where the experimental results seem to indicate a non-linearity that is not captured in the current methodology. The induced-drag effects of flap deflection were somewhat under-predicted; in contrast, moment-coefficient effects were notably over-predicted. For the landing flap setting, the predictions of the lift curve and drag polar compared well to the data, although the moment-coefficient increments were again over-predicted. For both the takeoff and landing flap settings, the predicted $C_{L_{\max }}$ compared well with the experimental data.

Plans exist to extend this methodology using higher-order aerodynamic methods such as a panel method and Euler analysis. Similar to the current methodology, empirical flap effectiveness factors can be used to define an effective flap deflection that takes into account the viscous effects without the need for a fullyviscous solution. The advantage of a full three-dimensional analysis is that it would enable the use of the pressure-difference rule for the prediction of $C_{L_{\max }}$, which has been shown to produce robust and accurate predictions of wing stall. ${ }^{20}$ The primary disadvantage of a three-dimensional analysis would be that the flap and slat surface shapes would need to be defined in detail, rather than the more basic information, such as flap chord fraction, that is needed to perform a vortex-lattice analysis.

\section{Acknowledgments}

This work was conducted as part of the NASA Aeronautical Sciences Project, led by James D. Heidmann, within the Multi-Disciplinary Design, Analysis and Optimization element, led by Jeffrey S. Robinson and Jeffrey K. Viken. The author wishes to thank Andrew S. Hahn and Mathias Wintzer for their technical and editorial assistance.

\section{References}

\footnotetext{
${ }^{1}$ Rumsey, C. L., Long, M., Stuever, R. A., and Wayman, T. R., "Summary of the First AIAA CFD High Lift Prediction Workshop (Invited)," 49th AIAA Aerospace Sciences Meeting including the New Horizons Forum and Aerospace Exposition, AIAA, Orlando, FL, 2011.

${ }^{2}$ Rumsey, C. L. and Slotnick, J. P., "Overview and Summary of the Second AIAA High Lift Prediction Workshop (Invited)," 52nd AIAA Aerospace Sciences Meeting, AIAA, National Harbor, MD, 2014.

${ }^{3}$ Raymer, D. P., Aircraft Design: A Conceptual Approach, AIAA Education Series, Washington, DC, 1989.

${ }^{4}$ Anon., "USAF Stability and Control DATCOM," ADB072483, Wright-Patterson Air Force Base, Ohio, 1976.

${ }^{5}$ XFoil, Software Package, Ver. 6.97, Massachusetts Institute of Technology, Cambridge, MA, 2008.
} 
${ }^{6}$ MSES, Software Package, Ver. 3.08, Massachusetts Institute of Technology, Cambridge, MA, 2008.

${ }^{7}$ Torenbeek, E., Synthesis of Subsonic Airplane Design, Kluwer Academic Publishers, Boston, 1982.

${ }^{8}$ Glauert, H., "Theoretical Relationships for an Aerofoil with Hinged Flap," ARC R and M no. 1095, Aeronautical Research Committee, April 1927.

${ }^{9}$ Foster, D. N., Ashill, P. R., and Williams, B. R., "The Nature, Development and Effect of the Viscous Flow Around an Aerofoil with High-Lift Devices," CP-1258, Her Majesty's Stationery Office, London, 1974

${ }^{10}$ Smith, A. M. O., "High-Lift Aerodynamics," Journal of Aircraft, Vol. 12, No. 6, 1975, pp. 501-530.

${ }^{11}$ AVL, Software Package, Ver. 3.32, Massachusetts Institute of Technology, Cambridge, MA, 2012.

12 FRICTION, Software Package, Ver. 3, Virginia Polytechnic Institute and State University, Blacksburg, VA, 2001.

${ }^{13}$ Wakayama, S. and Kroo, I., "Subsonic Wing Planform Design Using Multidisciplinary Optimization," Journal of Aircraft, Vol. 32, No. 4, 1995, pp. 746-753.

${ }^{14}$ Furlong, G. C. and McHugh, J. G., "A Summary and Analysis of the Low-Speed Longitudinal Characteristics of Swept Wings and High Reynolds Number," NACA report 1339, 1957.

${ }^{15}$ Omar, E., Zierten, T., and Mahal, A., "Two-Dimensional Wind-Tunnel Tests of a NASA Supercritical Airfoil with Various High-Lift Systems: Volume I Data Analysis," NASA CR-2214, April 1973.

${ }^{16}$ Omar, E., Zierten, T., Hahn, M., Szpiro, E., and Mahal, A., "Two-Dimensional Wind-Tunnel Tests of a NASA Supercritical Airfoil with Various High-Lift Systems: Volume II Test Data," NASA CR-2215, Sept. 1973.

${ }^{17}$ Morgan, H. L, Jr., "Model Geometry Description and Pressure Distribution Data from Tests of EET High-Lift Research Model Equipped with Full-Span Slat and Part-Span Flaps," NASA TM-80048, Feb. 1979.

${ }^{18}$ Morgan, H. L, Jr. and Paulson, J. W., "Low-Speed Aerodynamic Performance of a High-Aspect-Ratio Supercritical-Wing Transport Model Equipped with Full-Span Slat and Part-Span Double-Slotted Flaps," NASA TP-1580, Dec. 1979.

${ }^{19}$ Open VSP, Software Package, Ver. 2.2.5, NASA, 2013.

${ }^{20}$ Valarezo, W. O. and Chin, V. D., "Method for the Prediction of Wing Maximum Lift," Journal of Aircraft, Vol. 31, No. 1, 1994, pp. 103-109. 